\title{
Information Transfer between Generations Linked to Biodiversity in Rock-Paper-Scissors Games
}

\author{
Ranjan Bose \\ Department of Electrical Engineering, IIT Delhi, Hauz Khas, New Delhi 110016, India \\ Correspondence should be addressed to Ranjan Bose; rbose@ee.iitd.ac.in
}

Received 4 February 2015; Accepted 19 May 2015

Academic Editor: François Guerold

Copyright (C) 2015 Ranjan Bose. This is an open access article distributed under the Creative Commons Attribution License, which permits unrestricted use, distribution, and reproduction in any medium, provided the original work is properly cited.

Ecological processes, such as reproduction, mobility, and interaction between species, play important roles in the maintenance of biodiversity. Classically, the cyclic dominance of species has been modelled using the nonhierarchical interactions among competing species, represented by the "Rock-Paper-Scissors" (RPS) game. Here we propose a cascaded channel model for analyzing the existence of biodiversity in the RPS game. The transition between successive generations is modelled as communication of information over a noisy communication channel. The rate of transfer of information over successive generations is studied using mutual information and it is found that "greedy" information transfer between successive generations may lead to conditions for extinction. This generalized framework can be used to study biodiversity in any number of interacting species, ecosystems with unequal rates for different species, and also competitive networks.

\section{Introduction}

Ecological processes, such as reproduction, mobility, and interaction between species, have been shown to play important roles in the maintenance of biodiversity [1-5]. One of the useful methods for investigating codevelopment of populations is to use evolutionary game theory [6-8]. In this context, the Rock-Paper-Scissors (RPS) game is a paradigm to describe species diversity $[3,4]$. The cyclic dominance of species is modelled using the nonhierarchical interactions among competing species, represented by the "Rock-PaperScissors" (RPS) game in which rock crushes scissors, scissors cut paper, and paper wraps rock [9-16]. Communities of subpopulations or species exhibiting such dynamics have been identified in several ecosystems [17]. For example, cyclic dominance of three male strategies has been reported in lizards [18].

It has been shown that cyclic dominance alone is not sufficient to preserve biodiversity. When the interactions between individuals are local, spatially separated domains are dominated by one species form, leading to stable coexistence [1-3]. It is shown that beyond a critical value of mobility biodiversity is lost [10]. Researchers have shown that spiral formation takes place without a conservation law for the total density and, in general, fast diffusion can destroy species coexistence [11]. The final survival probabilities characterizing different species in cyclic competition have also been reported [12]. The interplay of evolutionary dynamics, discreteness of the population, and the nature of the interactions also influence the coexistence of strategies [13]. Pattern formations due to cyclic dominance of three species, operating near a bifurcation point, have also been investigated [14]. The spreading of epidemic and its effect on biodiversity has been studied using the RPS game [15]. The role of swarming in the context of biodiversity has also been investigated [16]. A recently published review paper on the cyclic dominance in evolutionary games covers in detail the current advances on RPS and related evolutionary games. In particular, it focuses on pattern formation, the impact of mobility, and the spontaneous emergence of cyclic dominance. The authors also review mean-field and zerodimensional RPS models and the application of the complex Ginzburg-Landau equation and highlight the importance of statistical physics for the study of large-scale ecological systems [19]. Theoretical explorations of the relevance of spatial structure in evolutionary games have also received a lot of attention in the recent past [20-22]. Realistic models, beyond 
well-mixed populations, have been studied recently [23, 24]. Researchers have also used mean-field theory to show that group interactions at the mesoscopic scale have been taken into account for the coexistence of a large number of species [25]. Recent work on mobility and velocity-enhanced cooperation of moving agents has further linked mobility to biodiversity $[26,27]$. The Ginzburg-Landau theory has also been applied successfully to study biodiversity using models of spatial RPS games [28, 29]. Investigation of alliance formation among multiple number of interacting species shows that stable solutions are possible, depending on the parameter values that determine invasion and mixing in the multispecies predator-prey systems [30].

The primary motivation of this work is to model the information transfer between successive generations of evolution and link it to biodiversity. Further, we wish to explore the effect of the rate of information transfer on the existence of biodiversity. Yet another motivation is to study the nonhierarchical cyclic interactions between $N$ species using this information-theoretic approach. In this paper, we provide a novel approach using a cascaded communication channel for analyzing biodiversity in the classic Lotka-Volterra model. Specifically, we investigate the transitions from one generation to the next based on the transition probability matrix between two consecutive generations. The transition between successive generations is modelled as communication of information through a noisy communication channel. We derive explicit relation between the probabilities of the species in two consecutive generations in terms of the three basic processes: selection, reproduction, and mobility. This gives us an elegant method to study the evolution of the species for long waiting times and ultimately leads to the condition for the existence of biodiversity. We also show how selection, reproduction, and mobility together influence the existence of biodiversity.

The paper is organized as follows. Section 1 is the Introduction. In Section 2, the system model is discussed in detail. In Section 3, the transition from one generation to the next is modelled as a problem of communicating symbols across a noisy channel. The information transfer across generations is linked to the existence of biodiversity in Section 4 . The proposed method is generalized for $N$ species in Section 5 . Results from lattice simulations are presented in Section 6. Finally, the paper is concluded in Section 7.

\section{System Model}

We consider the cyclic Lotka-Volterra model where three states $A, B$, and $C$ cyclically dominate each other [31]. Let the time densities for the three species be $a(t), b(t)$, and $c(t)$, respectively. The deterministic rate equations describing the time evolution of these three species can be written as

$$
\begin{aligned}
& \dot{a}=a\left(k_{C} b-k_{B} c\right), \\
& \dot{b}=b\left(k_{A} c-k_{C} a\right), \\
& \dot{c}=c\left(k_{B} a-k_{A} b\right) .
\end{aligned}
$$

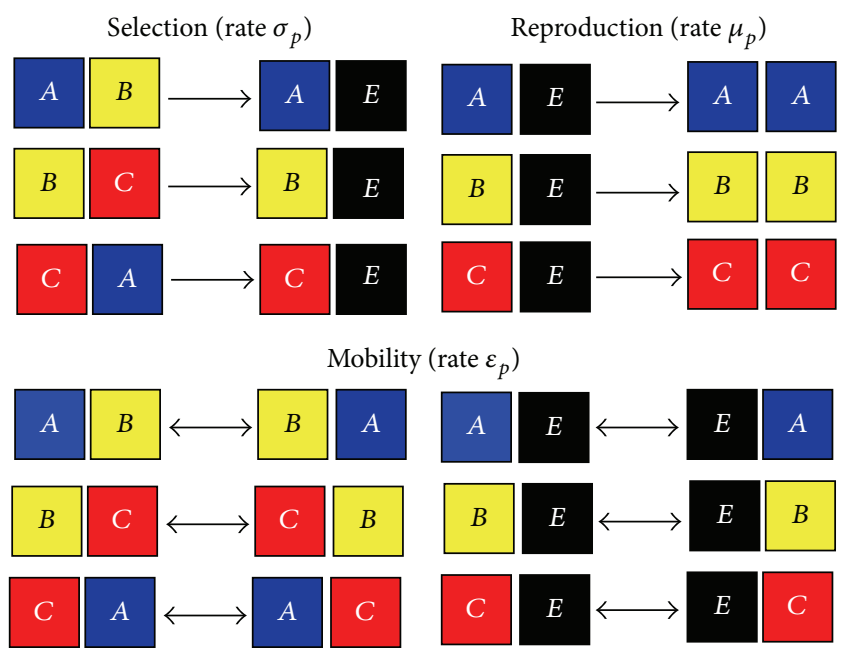

FIgure 1: The stochastic model. The individuals of the three species are represented by $A$ (blue), $B$ (yellow), and $C$ (red). The individuals interact only with their immediate neighbors. The selection, reproduction, and mobility are modelled as the Poisson processes with rates $\sigma_{p}, \mu_{p}$, and $\varepsilon_{p}$, respectively.

As the spatial version of the Lotka-Volterra model, we consider three subpopulations or species $(A, B$, and $C)$, the members of which are distributed randomly over an $M \times$ $M$ spatial lattice. There are also empty spaces $(E)$ on the lattice, which are essentially unoccupied. For the possible interactions with the neighbours, we consider a version of the RPS game, namely, a stochastic spatial variant of the model [4]. The Rock, Paper, and Scissors are depicted by $A, B$, and $C$, respectively. Figure 1 shows the basic processes of selection, reproduction, and mobility, modelled as the Poisson processes, occurring at rates $\sigma_{p}, \mu_{p}$, and $\varepsilon_{p}$, respectively. The individuals interact only with their immediate neighbors.

\section{Modeling Transition between Generations}

Let us model the transition from generation $i$ to $i+1$ as a problem of communicating four types of symbols $(A, B, C$, and $E$ ) across a noisy channel. This channel occasionally makes errors which result in one symbol being received as a different symbol. This is similar to a standard communication channel problem encountered in electrical engineering [32]. This channel model is shown in Figure 2. In our case, the symbols are the lattice locations occupied by the individuals of the three species (or the empty locations). In the case of transition from one generation to the next, a site in the lattice currently occupied by a particular species may become occupied by an individual from another species due to mobility or fall vacant due to selection. Alternatively, an empty site may become occupied by a member from one of the species due to reproduction. It is also possible that there is no change in the occupancy of a lattice site. These transition probabilities are influenced by the selection rate, reproduction rate, and mobility.

The transition probabilities are based on averaged probabilities (densities) ignoring the spatial structure. This is 


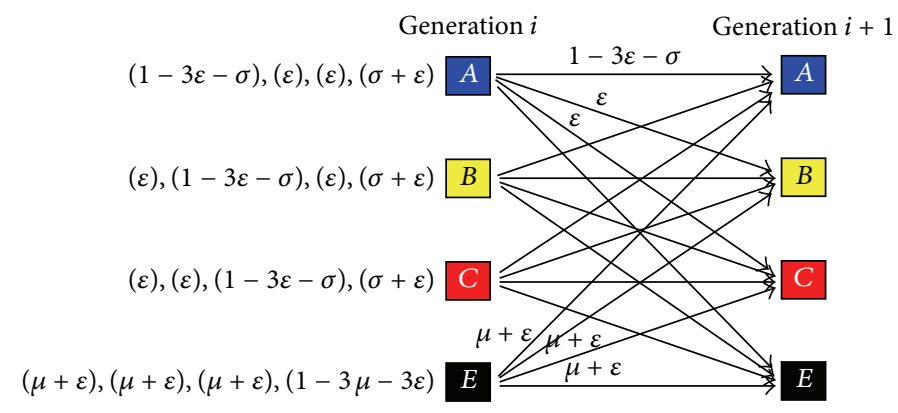

FIgURE 2: The channel model. The lattice locations occupied by the individuals of the three species undergo transition from one generation to the next. Instead of labelling all the arrows, the transition probabilities have been listed towards the left of the species, for clarity. Note that $\{\sigma, \mu, \varepsilon\}$ are the normalized rates.

a mean-field approach, assuming a well-mixed population with the limit of population size $N \rightarrow \infty$. The idea can be extended by considering joint densities of pairs of neighbours, and, to approximate higher order densities, one can consider products of lower order densities (so-called pair approximation and moment closure) [33].

The channel transition probability matrix, for a wellmixed population, can be written as

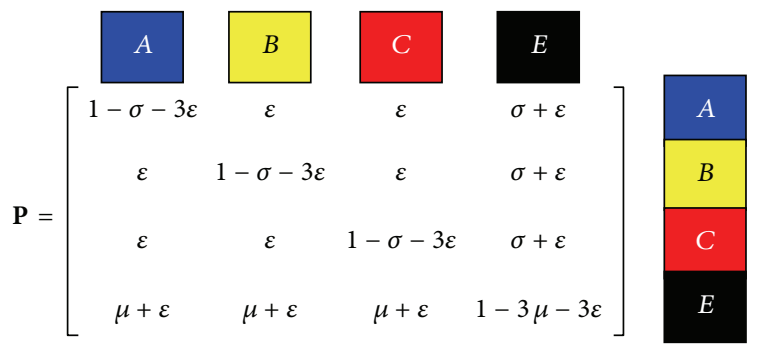

Any element $p_{l m}$ of the channel transition probability matrix represents the probability of transition of a particular site occupied by a member of species $l$ to the same site occupied by a member of species $m$ in the next generation. Here, $l, m=1,2,3$, and 4 correspond to $A, B, C$, and $E$, respectively. For example, $p_{12}$ represents the probability that the site occupied by species $A$ will become occupied by species $B$ in the subsequent generation. This is possible only by mobility, and hence $p_{12}=\varepsilon$. Similarly, $p_{34}=(\sigma+\varepsilon)$ represents the probability that the site occupied by species $C$ will translate to $E$ in the next generation. This is possible either by selection $(\sigma)$ or by mobility $(\varepsilon)$, which are mutually exclusive events, and hence the probabilities add up. It is important to note that the entries in the transition probability matrix $(\mathbf{P})$ are probabilities consisting of normalized $\{\sigma, \mu, \varepsilon\}$ such that each row adds up to unity. In order to differentiate, the rates have been denoted by $\sigma_{p}, \mu_{p}$, and $\varepsilon_{p}$.

From (2), the eigenvalues [34] of matrix $\mathbf{P}$ are found out to be $\lambda_{1}=1, \lambda_{2}=\lambda_{3}=1-4 \varepsilon-\sigma$, and $\lambda_{4}=1-4 \varepsilon-3 \mu-\sigma$. In the case of biodiversity, $P(A)=P(B)=P(C)$; that is, in the process of cyclic dominance, the occupation of the sites in the lattice by the three species is equiprobable. Let $p$ be the probability of occurrence of species $A, B$, or $C$ at a particular lattice site for generation $i$ and let $q$ be the probability of occurrence of species $A, B$, or $C$ at a particular lattice site for generation $i+1$. We can alternately write the transition probability matrix as $\mathbf{P}=\mathbf{V D V}^{-1}$, where $\mathbf{V}$ is the matrix of the eigenvectors of $\mathbf{P}$ and $\mathbf{D}$ is the diagonal matrix with the diagonal elements as the eigenvalues [34]. Thus, $\mathbf{P}$ can be written, in terms of the eigenvalues, as

$$
\mathbf{P}=\left[\begin{array}{cccc}
\alpha & \beta & \beta & \gamma \\
\beta & \alpha & \beta & \gamma \\
\beta & \beta & \alpha & \gamma \\
\delta & \delta & \delta & 1-3 \delta
\end{array}\right],
$$

where

$$
\begin{aligned}
\alpha & =\frac{1}{(\sigma+3 \mu+4 \varepsilon)}\left((\mu+\varepsilon)+\frac{2}{3}(\sigma+3 \mu+4 \varepsilon) \lambda_{2}\right. \\
& \left.+\frac{1}{3}(\sigma+\varepsilon) \lambda_{4}\right) \\
\beta & =\frac{1}{(\sigma+3 \mu+4 \varepsilon)}\left((\mu+\varepsilon)-\frac{1}{3}(\sigma+3 \mu+4 \varepsilon) \lambda_{2}\right. \\
& \left.+\frac{1}{3}(\sigma+\varepsilon) \lambda_{4}\right) \\
\gamma & =\frac{1}{(\sigma+3 \mu+4 \varepsilon)}\left((\sigma+\varepsilon)-(\sigma+\varepsilon) \lambda_{4}\right), \\
\delta & =\frac{1}{(\sigma+3 \mu+4 \varepsilon)}\left((\mu+\varepsilon)-(\mu+\varepsilon) \lambda_{4}\right) .
\end{aligned}
$$

We note that the transition probability matrices given in (2) and (3) are equivalent. Using (3) we can express the probabilities of individual species. For example, for species $A$, the next generation $A$ may result from $A, B, C$, or $E$, as dictated by the transition probability matrix given in (3). Thus, for species $A$, the probability of the next generation can be expressed as

$$
q=p \alpha+p \beta+p \beta+(1-3 p) \delta .
$$

Similar expressions can be derived for species $B$ and $C$. Solving (5), we obtain

$$
q=\left(\frac{\mu+\varepsilon}{\sigma+3 \mu+4 \varepsilon}\right)+\left(p-\left(\frac{\mu+\varepsilon}{\sigma+3 \mu+4 \varepsilon}\right)\right) \lambda_{4}
$$




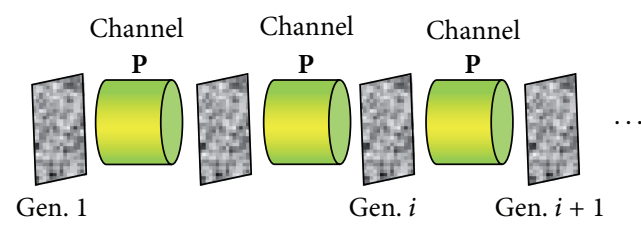

(a)

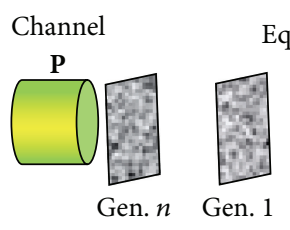

Equivalent channel,

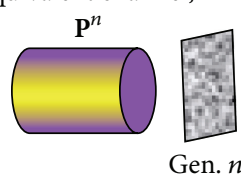

(b)

FIGURE 3: The cascaded channel model. (a) The transition from one generation to the next can be modelled as communication of information through a noisy channel. Thus, long waiting times can be modelled as a cascade of similar channels. (b) The equivalent channel for $n$ generations.

Equation (6) gives and explicit relation between the probabilities of species $p$ in generation $i$ to the probabilities of species, $q$, in the consecutive generation $i+1$.

\section{Link to Biodiversity}

So far we have considered two consecutive generations only. We now develop a mathematical framework for large waiting times, that is, for a large number of generations $(t \propto$ $M^{2}$ ). As we let the generations evolve using the stochastic spatial variant of the of the RPS game, it is equivalent to communicating through a cascade of noisy channels, as depicted in Figure 3(a). The equivalent channel transition probability matrix for $n$ cascaded channels [32] is given by $\mathbf{P}^{n}$. Since we can express $\mathbf{P}^{n}=\mathbf{V D}^{n} \mathbf{V}^{-1}$, the eigenvalues of $\mathbf{P}^{n}$ are given simply by $\left\{\lambda_{1}^{n}, \lambda_{2}^{n}, \lambda_{3}^{n}, \lambda_{4}^{n}\right\}$, where $\left\{\lambda_{1}, \lambda_{2}, \lambda_{3}, \lambda_{4}\right\}$ are the eigenvalues of $\mathbf{P}$. Thus, $n$ cascaded channels can be represented as one "equivalent channel," as shown in Figure 3(b). From (6), we can easily write the probabilities of species $A, B$, and $C$, after $n$ generations, as

$$
q_{n}=\left(\frac{\mu+\varepsilon}{\sigma+3 \mu+4 \varepsilon}\right)+\left(p-\left(\frac{\mu+\varepsilon}{\sigma+3 \mu+4 \varepsilon}\right)\right) \lambda_{4}^{n} .
$$

Since the matrix $\mathbf{P}$ is a probability transition matrix, $\left|\lambda_{4}\right|<\left|\lambda_{\max }\right|=1$ [22]. Thus, $\lambda_{4}^{n} \rightarrow 0$. Hence, from (7) we can conclude that a well-mixed population must always result in biodiversity.

We now consider the real-life scenario when the population is not well-mixed. In fact, clustering is routinely observed as the population evolves $[10,14,31]$. In this case, also the assumption of large population sizes exists; that is, size $N \rightarrow$ $\infty$. Let us represent the resultant transition matrix for a nonwell-mixed (clustered) scenario as

$$
\mathbf{R}_{i}=\mathbf{P}+\mathbf{Q}_{i}
$$

where $\mathbf{P}$ represents the transition matrix corresponding to a well-mixed population and $\mathbf{Q}_{i}$ represents the effect of local clustering at generation $i$. We observe that the effect of the $\mathbf{Q}_{i}$ matrix is to alter the transition probabilities (the branch labels of Figure 2). Since the resultant matrix, $\mathbf{R}_{i}$, is also a probability transition matrix with the individual rows adding up to unity, $\mathbf{Q}_{i}$ must be a matrix with individual rows adding up to zero. After $n$ generations, the effective channel can be represented by $\mathbf{R}_{\mathrm{eff}}=\prod_{i=1}^{n} \mathbf{R}_{i}$ where $\mathbf{R}_{i}$ is the resultant transition matrix for generation $i$. The relation between the probabilities for species $A, B$, and $C$ and the vacant locations, $E$, can be expressed as

$$
\left[\begin{array}{llll}
p_{A} & p_{B} & p_{C} & p_{E}
\end{array}\right]_{n}=\left[\begin{array}{llll}
p_{A} & p_{B} & p_{C} & p_{E}
\end{array}\right]_{1} \mathbf{R}_{\mathrm{eff}}
$$

where $\left[\begin{array}{llll}p_{A} & p_{B} & p_{C} & p_{E}\end{array}\right]_{n}$ represents the resulting probabilities for species $A, B$, and $C$ and the vacant locations, $E$, after $n$ generations, while $\left[\begin{array}{llll}p_{A} & p_{B} & p_{C} & p_{E}\end{array}\right]_{1}$ represents the starting probabilities. Extinction implies that one of the species dies out; that is, the left hand side of (9) should be $\left[\begin{array}{llll}0 & p_{B} & p_{C} & p_{E}\end{array}\right]_{n}$ or $\left[\begin{array}{llll}p_{A} & 0 & p_{C} & p_{E}\end{array}\right]_{n}$ or $\left[\begin{array}{lllll}p_{A} & p_{B} & 0 & p_{E}\end{array}\right]_{n}$ for arbitrary starting probabilities $\left[\begin{array}{llll}p_{A} & p_{B} & p_{C} & p_{E}\end{array}\right]_{1}$. This is possible if one of the first three columns of $\mathbf{R}_{\text {eff }}$ is zero, which gives us the necessary condition for extinction (uniformity), $\left|\lambda_{R_{\text {eff }}}\right|_{\min }=0$. Thus, the condition for the existence of biodiversity can be stated as

$$
\left|\lambda_{R_{\text {eff }}}\right|_{\min }>0
$$

Since $\mathbf{R}_{\mathrm{eff}}=\prod_{i=1}^{n} \mathbf{R}_{i}$, the condition for biodiversity can also be written as $\left|\lambda_{R_{i}}\right|_{\text {min }}>0$ for all $i$. This is one of our central results. An important contribution of this paper is to model the "transitions" of cells like the "transitions" of symbols over a noisy channel in communications. This leads to a matrix representation, which eventually has an elegant solution in terms of eigenvalues, when a cascade of channels is considered.

\section{Generalization to $N$ Species}

The given theory can be easily extended to study the nonhierarchical cyclic interactions between $N$ species in general. In this case, the two consecutive generations of $N$ interacting species can be linked using an $(N+1) \times(N+1)$ transition probability matrix with eigenvalues given by $\lambda_{1}=1, \lambda_{2}=$ $\lambda_{3}=\cdots=\lambda_{N}=1-\sigma-(N+1) \varepsilon$, and $\lambda_{N+1}=1-\sigma-N \mu-$ $(N+1) \varepsilon$. For a well-mixed population, the expression for the probabilities of $N$ species, after $n$ generations, is calculated to be

$$
\begin{aligned}
q_{n}= & \left(\frac{\mu+\varepsilon}{\sigma+N \mu+(N+1) \varepsilon}\right) \\
& +\left(p-\left(\frac{\mu+\varepsilon}{\sigma+N \mu+(N+1) \varepsilon}\right)\right) \lambda_{N+1}^{n} .
\end{aligned}
$$

We surmise from (11) that, in case of biodiversity, the lattice area occupied by each species, on an average, will be $((\mu+$ 


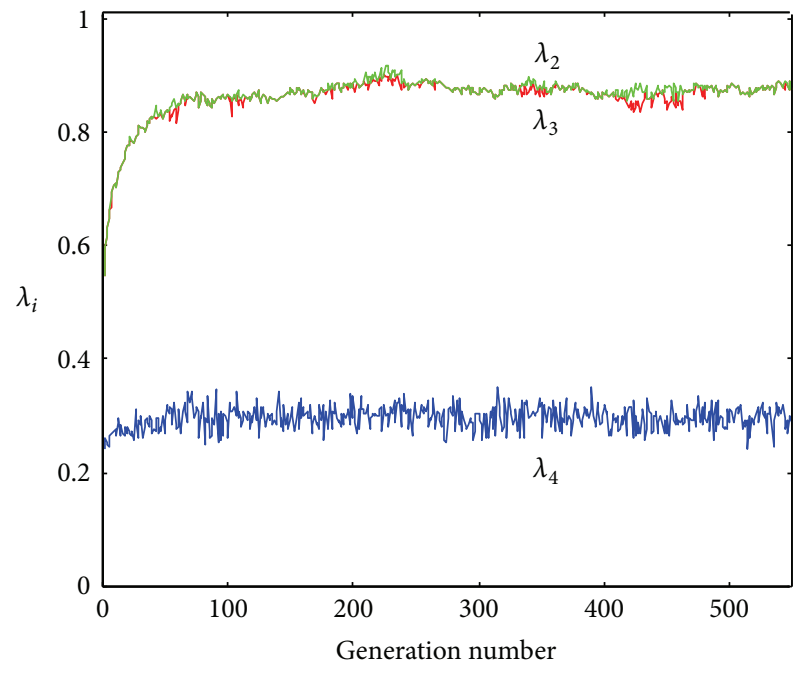

(a)

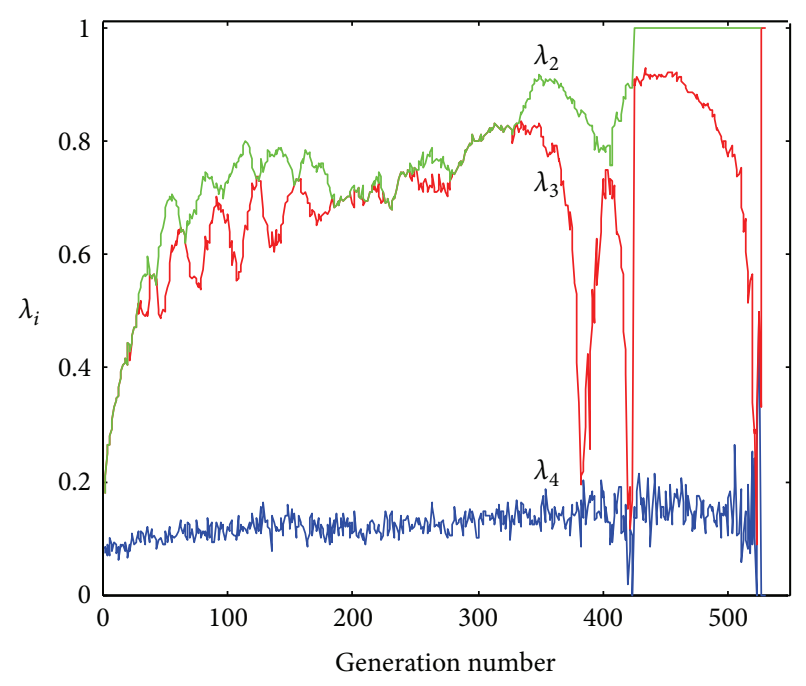

(b)

Figure 4: Typical plot of the eigenvalues of resultant probability matrix $\mathbf{R}_{i}$ where $i$ represents the generation number: $\lambda_{2}$ (red), $\lambda_{3}$ (green), and $\lambda_{4}$ (blue) (a) when biodiversity occurs and (b) when extinction happens.

$\varepsilon) /(\sigma+N \mu+(N+1) \varepsilon)) M^{2}$ where $M^{2}$ is the total number of lattice sites.

Another interesting aspect of cascaded channels is study of mutual information between consecutive generations. A discrete channel is a system with input alphabet $X$, output alphabet $Y$, and a probability transition matrix $p(y \mid x)$. The amount of information conveyed by the discrete channel is quantified by the mutual information between $X$ and $Y$ and is defined as [32]

$$
I(X ; Y)=H(Y)-H(Y \mid X),
$$

where $H(Y)$ is the entropy of $Y$ and $H(Y \mid X)$ is the entropy of $Y$ given $X$. The entropy, $H(Y)$ (in bits), is calculated using

$$
H(Y)=-\sum_{i=1}^{4} y_{i} \log _{2} y_{i}
$$

and, in general, $H(Z)=H\left(z_{1}, z_{2}, \ldots\right)=-\sum z_{i} \log _{2} z_{i}$, where $Z$ is a discrete random variable. The notion of mutual information is routinely used to determine the capacity of a channel in the area of communications. Here we wish to study how the rate of transfer of information over successive generations affects the possibility of coexistence among species. In our case, for a $4 \times 4 \mathbf{P}$ matrix, the expression for mutual information can be written as

$$
I(X ; Y)=\sum_{j=1}^{4} \sum_{i=1}^{4} P\left(y_{j}\right) P\left(x_{i} \mid y_{j}\right) \log \frac{P\left(x_{i} \mid y_{j}\right)}{P\left(x_{i}\right)} .
$$

Solving for mutual information, we obtain

$$
\begin{aligned}
I & (X ; Y) \\
= & H(Y) \\
& \quad-(3 p H(\alpha, \beta, \beta, \gamma)+(1-3 p) H(\delta, \delta, \delta, 1-3 \delta)),
\end{aligned}
$$

where $\alpha, \beta, \gamma$, and $\delta$ are given in (4).

\section{Lattice Simulations}

Extensive computer simulations were carried out to test the condition for biodiversity, as predicted by (10). Cyclic dominance is modeled using a stochastic lattice $[2,4]$. In our stochastic lattice simulations, we have arranged the three species on a two-dimensional square lattice with periodic boundary conditions. By periodic boundary conditions, we imply that the sites on the left edge of the grid are viewed as adjacent to ones on the right edge and those on the bottom are viewed as adjacent to those on the top. Every lattice site is occupied by an individual of species $A$, species $B$, or species $C$ or left empty $(E)$. At each simulation step, a random individual interacts with one of its eight nearest neighbours (corresponding to the move of the King in a chess game). The choice of the neighbour is also randomly determined (each of the eight neighbours are equally likely). The interaction with the neighbour, reproduction or migration, as well as the corresponding waiting time, is carried out according to a built-in function in MATLAB that generates random values from a Poisson distribution. In our simulations, one generation is counted when every individual has reacted, on an average, once.

Figure 4(a) shows a typical plot of the eigenvalues of $\mathbf{R}_{i}$ : $\lambda_{2}$ (red), $\lambda_{3}$ (green), and $\lambda_{4}$ (blue) when biodiversity occurs. Here the lattice size is $100 \times 100$. It is clear from the plot that $\left.\lambda_{R_{i}}\right|_{\text {min }}=\lambda_{4}>0$, and hence biodiversity is observed. In Figure 4(b), we show the case when extinction happens. Here, as soon as $\lambda_{4} \approx 0$, one of the species dies out.

Simulations were also carried out for different values of $\sigma_{p}, \mu_{p}$, and $\varepsilon_{p}$, and the system was tested for the existence of biodiversity (or uniformity) after long waiting periods. These operating points are plotted in Figure 5(a). We show the results for different system sizes: $50 \times 50$ lattice sites, $70 \times 70$ lattice sites, and $100 \times 100$ lattice sites. To obtain the extinction probability, the results are averaged over 100 


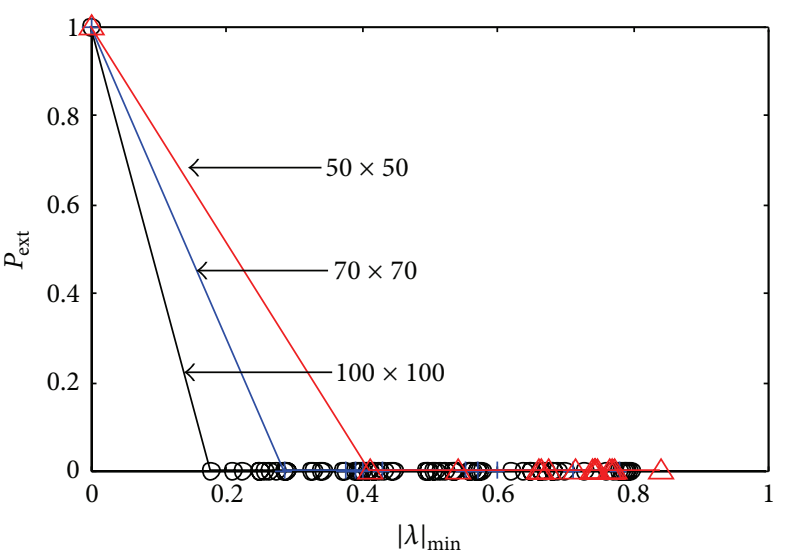

(a)

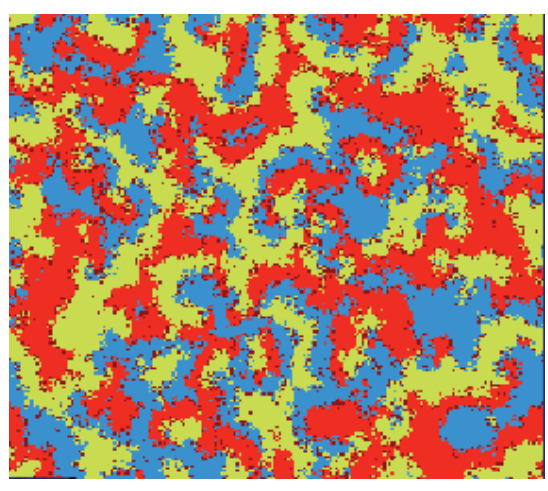

(b)

Figure 5: The probability of extinction, $P_{\text {ext }}$ (a) $P_{\text {ext }}$ for different system sizes: $50 \times 50$ (red $\triangle$ ), $70 \times 70$ (blue + ), and $100 \times 100$ (black o). (b) A typical snapshot from lattice simulations after a long waiting time for the case when biodiversity exists (three colors: $A, B$, and $C$, black dots: empty spaces, $E$ ).

realizations. Simulation results corroborate the theory. The probability of extinction, $P_{\text {ext }}$, tends to zero for the cases where $\left|\lambda_{R_{\text {eff }}}\right|_{\text {min }}>0$, while $\left|\lambda_{R_{i}}\right|_{\text {min }}=0$ leads to uniformity. We observe that as the system size increases, the transition between biodiversity and uniformity sharpens. In order to determine $\left|\lambda_{R_{i}}\right|$, the actual values of the normalized rates $\sigma, \mu$, and $\varepsilon$ were extracted by carrying out a frequency count of the number of lattice-site transitions from one generation to the next and then averaging these values over 500 generations. Figure 5(b) shows a typical snapshot from lattice simulations after a long waiting time for the case when biodiversity exists.

The proposed mathematical framework can be conveniently used to study systems with unequal reaction rates by appropriately modifying the transition matrix, $\mathbf{P}$. Suppose the three species have different normalized rates for selection, reproduction, and mobility, given by $\left\{\sigma_{i}, \mu_{i}, \varepsilon_{i}\right\}, i=1,2$, and 3. Then the corresponding transition probability matrix, for unequal normalized rates, can be written as

$$
\begin{aligned}
& \mathbf{P}_{U} \\
& =\left[\begin{array}{cccc}
1-\sigma_{3}-3 \varepsilon_{1} & \varepsilon_{1} & \varepsilon_{1} & \sigma_{3}+\varepsilon_{1} \\
\varepsilon_{2} & 1-\sigma_{1}-3 \varepsilon_{2} & \varepsilon_{2} & \sigma_{1}+\varepsilon_{2} \\
\varepsilon_{3} & \varepsilon_{3} & 1-\sigma_{2}-3 \varepsilon_{3} & \sigma_{2}+\varepsilon_{3} \\
\mu_{1}+\varepsilon_{1} & \mu_{2}+\varepsilon_{2} & \mu_{3}+\varepsilon_{3} & 1-\sum_{i=1}^{3}\left(\rho_{i}+\varepsilon_{i}\right)
\end{array}\right] .
\end{aligned}
$$

The eigenvalues of this generalized transition probability matrix can be used to study the effects of the different parameters on biodiversity. As an illustrative example, suppose we wish to investigate the effect of unequal (normalized) reproduction rates $\mu_{1}, \mu_{2}$, and $\mu_{3}$ for the three species ( $\sigma$ and $\varepsilon$ are the same for all three species). From (12) we can calculate the eigenvalues as $\lambda_{1}=1, \lambda_{2}=\lambda_{3}=1-\sigma-4 \varepsilon$, and $\lambda_{4}=1-\sigma-\left(\mu_{1}+\mu_{2}+\mu_{3}\right)-4 \varepsilon$.

Another interesting observation from nature is the fact that resource competitors can benefit one another through containment of shared competitors $[35,36]$. When several factors determine the outcome of competition, the interacting species can be modeled as a competitive network, as shown in Figure 6(a). Here, species are shown as nodes, and arrows connect the competitive inferior to the superior competitor. Researchers have shown that intransitivity in competitive networks can maintain diversity [3, 37]. The proposed cascaded channel model can be easily adapted to include different numbers of competitive relationships in a competitive network, as illustrated in Figure 6(a). Figure 6(b) gives the generic channel model for $r$ species and $s$ competitive relationships which can be represented as a transition probability matrix and analyzed for the existence of biodiversity as discussed earlier.

Figure 7 shows the plot of mutual information versus generation number for typical cases where coexistence is observed (cases (a) and (b)) and typical cases where extinction occurs (cases (c) and (d)). These simulations are for lattice size $100 \times 100$. In these experiments, for different sets of values for $\{\sigma, \mu, \varepsilon\}$, we determine whether the lattice simulations lead to biodiversity or uniformity. It is interesting to observe that $I(X ; Y)$ between successive generations is fairly stable for the cases when biodiversity occurs, while it shows an increasing trend for the cases when uniformity occurs. The trend is depicted by the dotted line in the figure. It appears that "greedy" information transfer between successive generations may lead to conditions for extinction.

\section{Conclusion}

We have developed a framework using cascaded channel model for analyzing the existence of biodiversity in nonhierarchical interactions among $N$ competing species. This mathematical framework gives a clear insight into how the three parameters, reproduction rate, selection rate, and mobility, influence biodiversity. To give a biological interpretation, we can draw a parallel to colicinogenic strains of $E$. coli growing on a Petri dish [3]. These strains can produce a toxin and the "antidote" which determine the selection rate $\left(\sigma_{p}\right)$. 


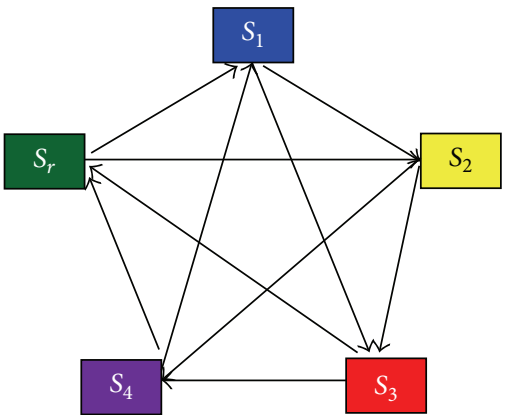

(a)

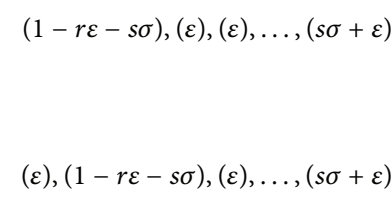

$(\varepsilon),(\varepsilon), \ldots,(1-r \varepsilon-s \sigma),(s \sigma+\varepsilon)$

$(s \mu+\varepsilon),(s \mu+\varepsilon),(s \mu+\varepsilon), \ldots,(1-r s \mu-r \varepsilon)$

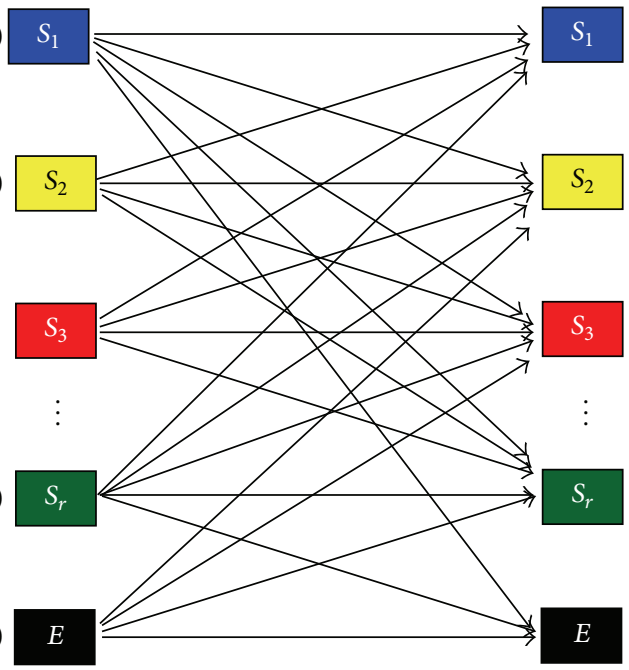

(b)

FiguRe 6: (a) Competitive network with $r$ species $\left(S_{1}, S_{2}, \ldots, S_{r}\right), r=5$, and $s=2$ competitive relationships. (b) Generic channel model with $r$ species and $s$ competitive relationships. Note that it is a fully connected channel model; however, not all connections are shown for the sake of clarity.

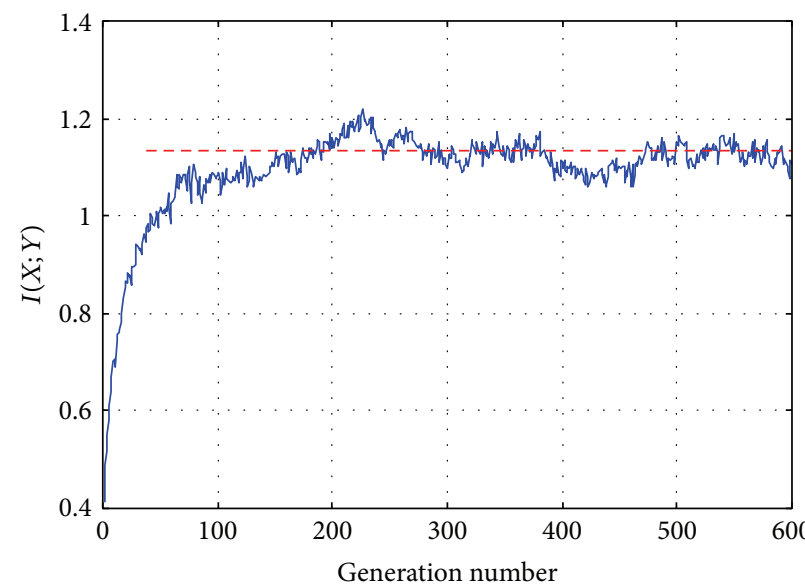

(a)

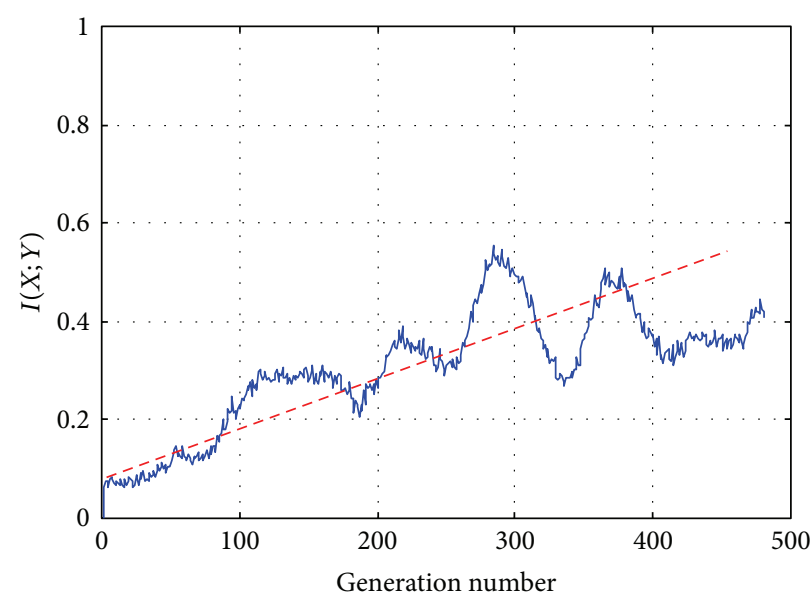

(c)

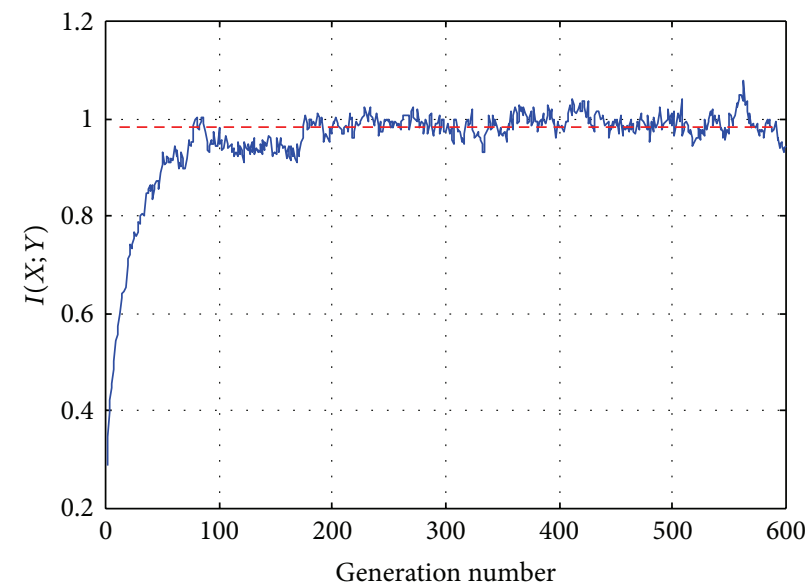

(b)

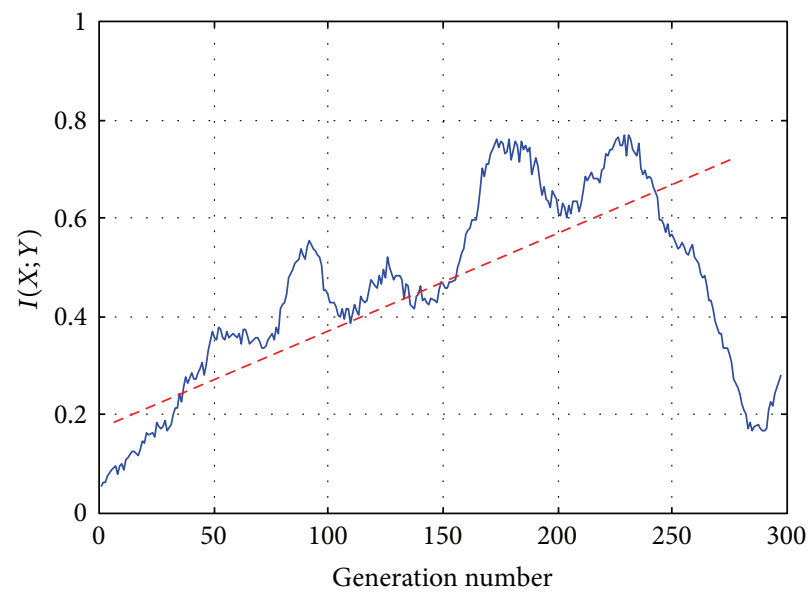

(d)

Figure 7: Mutual information, $I(X ; Y)$, between each consecutive generation for all generations. Cases (a) and (b) represent the typical scenarios which lead to biodiversity while (c) and (d) represent the cases when extinction occurs. Simulations are for lattice size $100 \times 100$. 
However, both the toxin and the "antidote" are generated by using resources and hence at the cost of their reproduction rate $\left(\mu_{p}\right)$. The mobility of $E$. coli can be increased by using supersoft agar. On the other hand, this mobility can be lowered by increasing the agar concentration. This is modeled by our mobility parameter $\varepsilon_{p}$.

The significant contributions of this paper are as follows.

(1) The transition between successive generations is modelled as communication of information through a noisy communication channel. This information theoretic model is a paradigm shift and is not linked to any of the previously reported models. For example, using this model one can understand the interdependence on reproduction rate, selection rate, and mobility together on the existence of biodiversity.

(2) The cascaded channel model exploits a very unique characteristic of matrices: the ability to express square matrices in terms of their eigenvalues. This approach permits a cascade of channels to be modelled as a product of several matrices. This approach leads to an elegant solution in terms of the power of the eigenvalues.

(3) The versatility of this approach allows it to be extended to study more than three interacting species, species with ecosystems with unequal rates for different species and competitive networks with different competitive relationships.

(4) The rate of transfer of information over successive generations is studied using mutual information, and it is found that "greedy" information transfer between successive generations may lead to conditions for extinction.

(5) Explicit dependence of the probability of population distribution on the transition probabilities linked to the reproduction rate, selection rate, and mobility is derived in this paper. The condition for biodiversity is derived, which is corroborated by simulations.

(6) This generalized mathematical framework can be used to study biodiversity in any number of interacting species, ecosystems with unequal rates for different species, and also competitive networks.

\section{Conflict of Interests}

The author declares that there is no conflict of interests regarding the publication of this paper.

\section{References}

[1] R. Durrett and S. Levin, "Allelopathy in spatially distributed populations," Journal of Theoretical Biology, vol. 185, no. 2, pp. 165-171, 1997.

[2] R. Durrett and S. Levin, "Spatial aspects of interspecific competition," Theoretical Population Biology, vol. 53, no. 1, pp. 30-43, 1998.

[3] B. Kerr, M. A. Riley, M. W. Feldman, and B. J. M. Bohannan, "Local dispersal promotes biodiversity in a real-life game of rock-paper-scissors," Nature, vol. 418, no. 6894, pp. 171-174, 2002.

[4] R. M. May and W. J. Leonard, "Nonlinear aspects of competition between three species," SIAM Journal on Applied Mathematics, vol. 29, no. 2, pp. 243-253, 1975.

[5] R. V. Solé and J. Bascompte, Self-Organization in Complex Ecosystems, Princeton University Press, Princeton, NJ, USA, 2006.

[6] J. von Neumann and O. Morgenstern, Theory of Games and Economic Behavior, Princeton University Press, Princeton, NJ, USA, 1944.

[7] J. M. Smith, Evolution and the Theory of Games, Cambridge University Press, 1982.

[8] J. W. Weibull, Evolutionary Game Theory, The MIT Press, Cambridge, Mass, USA, 2002.

[9] D. Neal, Introduction to Population Biology, Cambridge University Press, Cambridge, UK, 2004.

[10] T. Reichenbach, M. Mobilia, and E. Frey, "Mobility promotes and jeopardizes biodiversity in rock-paper-scissors games," Nature, vol. 448, no. 7157, pp. 1046-1049, 2007.

[11] M. Peltomäki and M. Alava, “Three- and four-state rock-paperscissors games with diffusion," Physical Review E: Statistical, Nonlinear, and Soft Matter Physics, vol. 78, no. 3, Article ID 031906, 7 pages, 2008.

[12] M. Berr, T. Reichenbach, M. Schottenloher, and E. Frey, "Zeroone survival behavior of cyclically competing species," Physical Review Letters, vol. 102, no. 4, Article ID 048102, 2009.

[13] J. C. Claussen and A. Traulsen, "Cyclic dominance and biodiversity in well-mixed populations," Physical Review Letters, vol. 100, no. 5, Article ID 058104, 2008.

[14] T. Reichenbach and E. Frey, "Instability of spatial patterns and its ambiguous impact on species diversity," Physical Review Letters, vol. 101, no. 5, Article ID 058102, 2008.

[15] W.-X. Wang, Y.-C. Lai, and C. Grebogi, "Effect of epidemic spreading on species coexistence in spatial rock-paper-scissors games," Physical Review E, vol. 81, no. 4, Article ID 046113, 2010.

[16] R. Bose, "Effect of swarming on biodiversity in non-symmetric rock-paper-scissor game," IET Systems Biology, vol. 4, no. 3, pp. 177-184, 2010.

[17] J. B. C. Jackson and L. Buss, "Allelopathy and spatial competition among coral reef invertebrates," Proceedings of the National Academy of Sciences of the United States of America, vol. 72, pp. 5160-5163, 1975.

[18] B. Sinervo and C. M. Lively, "The rock-scissors-paper game and the evolution of alternative male strategies," Nature, vol. 380, no. 6571, pp. 240-243, 1996.

[19] A. Szolnoki, M. Mobilia, L. Jiang, B. Szczesny, A. M. Rucklidge, and M. Perc, "Cyclic dominance in evolutionary games: a review," Journal of the Royal Society Interface, vol. 11, no. 100, Article ID 20140735, 2014.

[20] M. Perc and A. Szolnoki, "Coevolutionary games: a mini review," BioSystems, vol. 99, no. 2, pp. 109-125, 2010.

[21] E. Frey, "Evolutionary game theory: theoretical concepts and applications to microbial communities," Physica A, vol. 389, no. 20, pp. 4265-4298, 2010.

[22] M. Perc, J. Gómez-Gardeñes, A. Szolnoki, L. M. Floría, and Y. Moreno, "Evolutionary dynamics of group interactions on structured populations: a review," Journal of The Royal Society Interface, vol. 10, no. 80, Article ID 20120997, 2013. 
[23] P. Bednarik, K. Fehl, and D. Semmann, "Costs for switching partners reduce network dynamics but not cooperative behaviour," Proceedings of the Royal Society B: Biological Sciences, vol. 281, no. 1792, Article ID 20141661, 2014.

[24] G. S. van Doorn, T. Riebli, and M. Taborsky, "Coaction versus reciprocity in continuous-time models of cooperation," Journal of Theoretical Biology, vol. 356, pp. 1-10, 2014.

[25] H. Cheng, N. Yao, Z. Huang, J. Park, Y. Do, and Y. Lai, "Mesoscopic interactions and species coexistence in evolutionary game dynamics of cyclic competitions," Scientific Reports, vol. 4, article 7486, 2014.

[26] A. Cardillo, S. Meloni, J. Gómez-Gardeñes, and Y. Moreno, "Velocity-enhanced cooperation of moving agents playing public goods games," Physical Review E, vol. 85, Article ID 067101, 2012.

[27] M. H. Vainstein and J. J. Arenzon, "Spatial social dilemmas: dilution, mobility and grouping effects with imitation dynamics," Physica A, vol. 394, pp. 145-157, 2014.

[28] I. S. Aranson and L. Kramer, "The world of the complex Ginzburg-Landau equation," Reviews of Modern Physics, vol. 74, no. 1, pp. 99-143, 2002.

[29] B. Szczesny, M. Mobilia, and A. Rucklidge, "Characterization of spiraling patterns in spatial rock-paper-scissors games," Physical Review E, vol. 90, Article ID 032704, 2014.

[30] G. Szabó, A. Szolnoki, and I. Borsos, "Self-organizing patterns maintained by competing associations in a six-species predatorprey model," Physical Review E, vol. 77, no. 4, Article ID 041919, 2008.

[31] T. Reichenbach, M. Mobilia, and E. Frey, "Self-organization of mobile populations in cyclic competition," Journal of Theoretical Biology, vol. 254, no. 2, pp. 368-383, 2008.

[32] R. Bose, Information Theory, Coding and Cryptography, Tata McGraw-Hill, Noida, India, 2002.

[33] G. Szabó and G. Fáth, "Evolutionary games on graphs," Physics Reports, vol. 446, no. 4-6, pp. 97-216, 2007.

[34] R. A. Horn and C. R. Johnson, Matrix Analysis, Cambridge University Press, Cambridge, UK, 2012.

[35] L. Stone and A. Roberts, "Conditions for a species to gain advantage from the presence of competitors," Ecology, vol. 72, no. 6, pp. 1964-1972, 1991.

[36] S. Allesina and J. M. Levine, "A competitive network theory of species diversity," Proceedings of the National Academy of Sciences of the United States of America, vol. 108, no. 14, pp. 56385642, 2011.

[37] R. A. Laird and B. S. Schamp, "Competitive intransitivity promotes species coexistence," The American Naturalist, vol. 168, no. 2, pp. 182-193, 2006. 

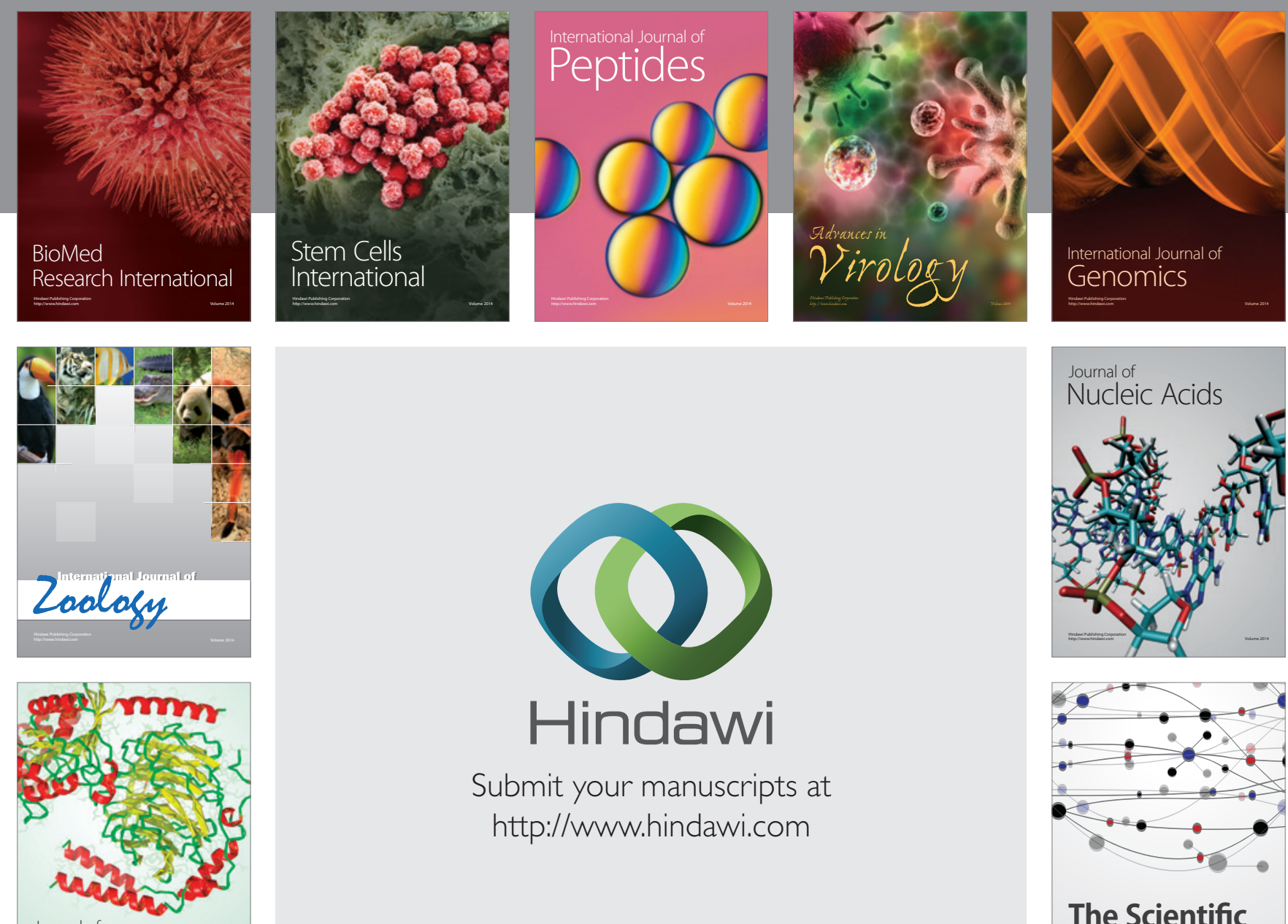

Submit your manuscripts at

http://www.hindawi.com

Journal of
Signal Transduction
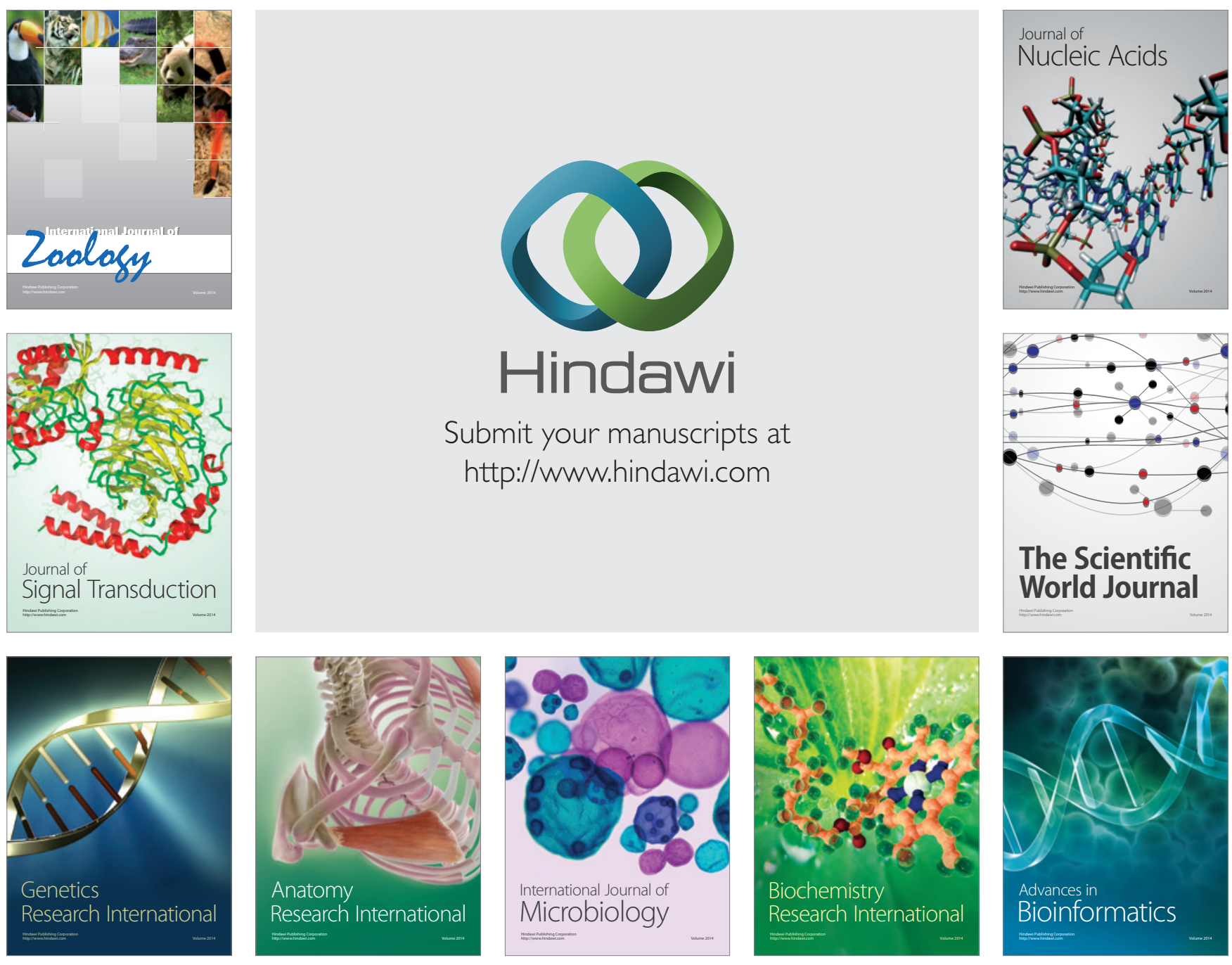

The Scientific World Journal
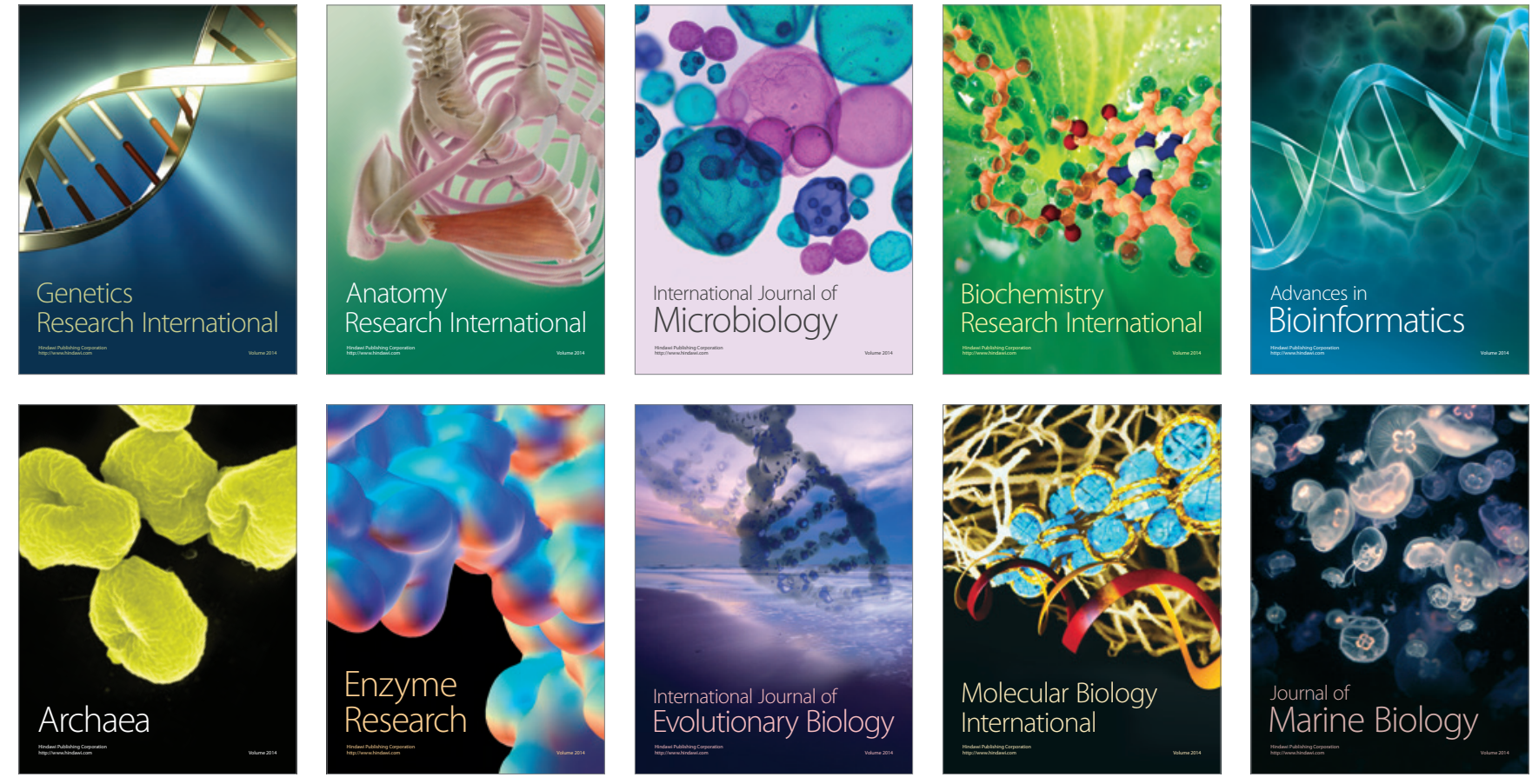